\title{
Quantile Regression and Beyond in Statistical Analysis of Data
}

\author{
Rahim Alhamzawi $\mathbb{D}^{1},{ }^{1}$ Keming Yu, ${ }^{2}$ and Himel Mallick $\mathbb{D}^{3}$ \\ ${ }^{1}$ Department of Statistics, University of Al-Qadisiyah, Al-Qadisiyah, Iraq \\ ${ }^{2}$ Brunel University, Uxbridge, UK \\ ${ }^{3}$ Harvard University, Boston, USA \\ Correspondence should be addressed to Rahim Alhamzawi; ralhamzawi@yahoo.com
}

Received 11 June 2019; Accepted 11 June 2019; Published 22 July 2019

Copyright (c) 2019 Rahim Alhamzawi et al. This is an open access article distributed under the Creative Commons Attribution License, which permits unrestricted use, distribution, and reproduction in any medium, provided the original work is properly cited.

Regression is used to quantify the relationship between response variables and some covariates of interest. Standard mean regression has been one of the most applied statistical methods for many decades. It aims to estimate the conditional expectation of the response variable given the covariates. However, quantile regression is desired if conditional quantile functions such as median regression are of interest. Quantile regression has emerged as a useful supplement to standard mean regression. Also, unlike mean regression, quantile regression is robust to outliers in observations and makes very minimal assumptions on the error distribution and thus is able to accommodate nonnormal errors. The value of "going beyond the standard mean regression" has been illustrated in many scientific subjects including economics, ecology, education, finance, survival analysis, microarray study, growth charts, and so on. In addition, inference on quantiles can accommodate transformation of the outcome of the interest without the problems encountered in standard mean regression. Overall, quantile regression offers a more complete statistical model than standard mean regression and now has widespread applications.

There has been a great deal of recent interest in Bayesian approaches to quantile regression models and the applications of these models. In these approaches, uncertain parameters are assigned prior distributions based on expert judgment and updated using observations through the Bayes formula to obtain posterior probability distributions. In this special issue on "Quantile regression and beyond in statistical analysis of data," we have invited a few papers that address such issues. The first paper of this special issue addresses a fully Bayesian approach that estimates multiple quantile levels simultaneously in one step by using the asymmetric Laplace distribution for the errors, which can be viewed as a mixture of an exponential and a scaled normal distribution. This method enables characterizing the likelihood function by all quantile levels of interest using the relation between two distinct quantile levels. The second paper presents a new link function for distribution-specific quantile regression based on vector generalized linear and additive models to directly model specified quantile levels. The third paper presents a novel modeling approach to study the effect of predictors of various types on the conditional distribution of the response variable. The fourth paper introduces the regularized quantile regression method using pairwise absolute clustering and sparsity penalty, extending from mean regression to quantile regression setting. The final paper of this special issue uses Bayesian quantile regression for studying the retirement consumption puzzle, which is defined as the drop in consumption upon retirement, using the cross-sectional data of the Malaysian Household Expenditure Survey 2009/2010.

\section{Conflicts of Interest}

The authors declare that the research was conducted in the absence of any commercial or financial relationships that could be construed as potential conflicts of interest.

Rahim Alhamzawi Keming $\mathrm{Yu}$ Himel Mallick 


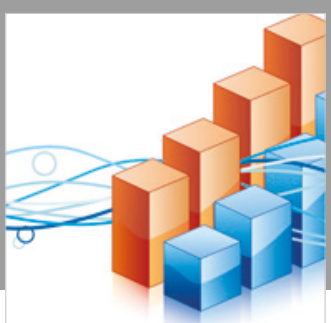

Advances in

Operations Research

\section{-n-m}
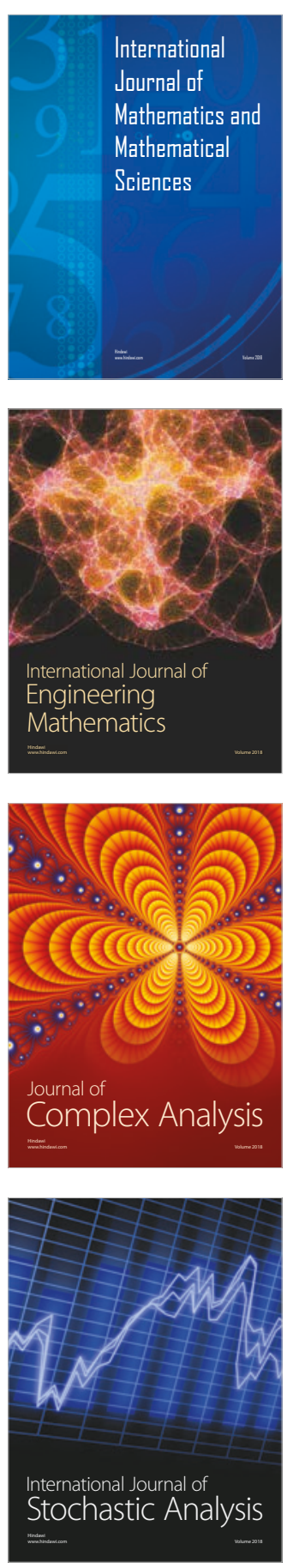
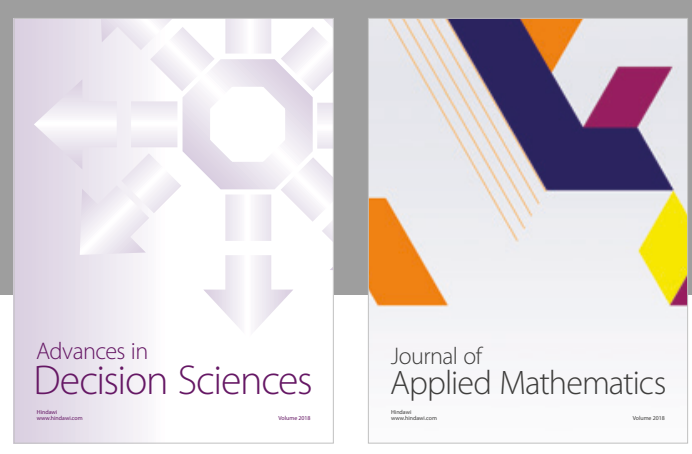

Journal of

Applied Mathematics
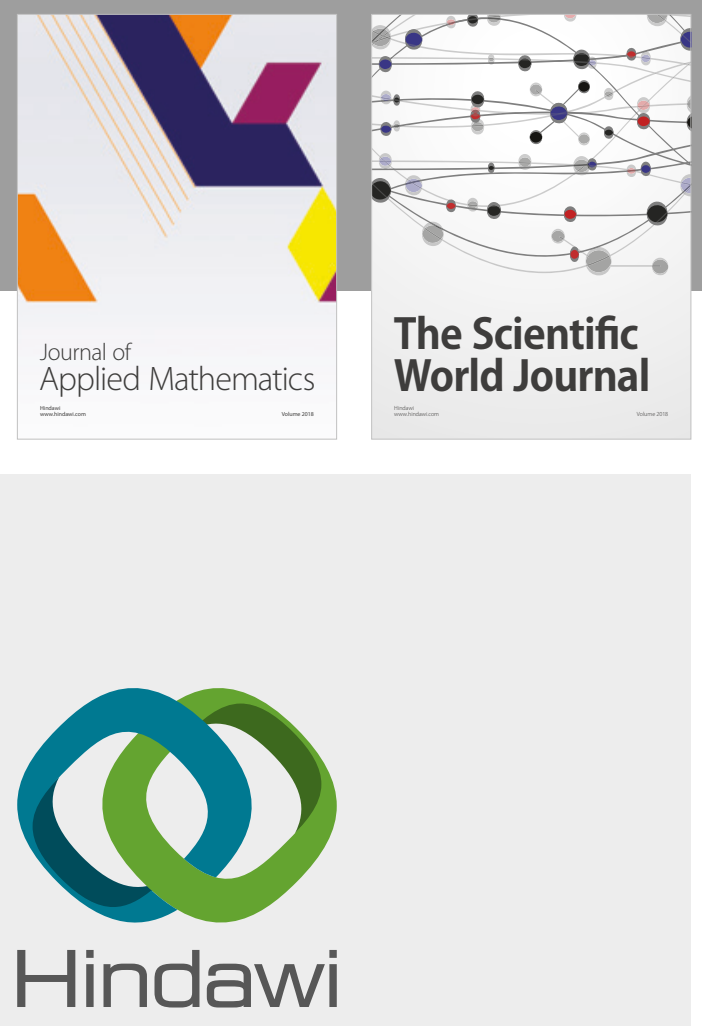

Submit your manuscripts at

www.hindawi.com

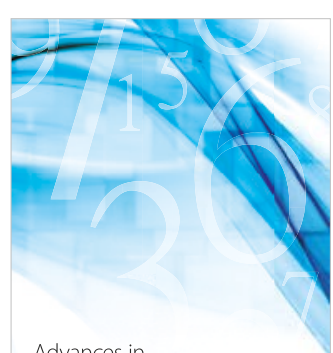

Advances in
Numerical Analysis
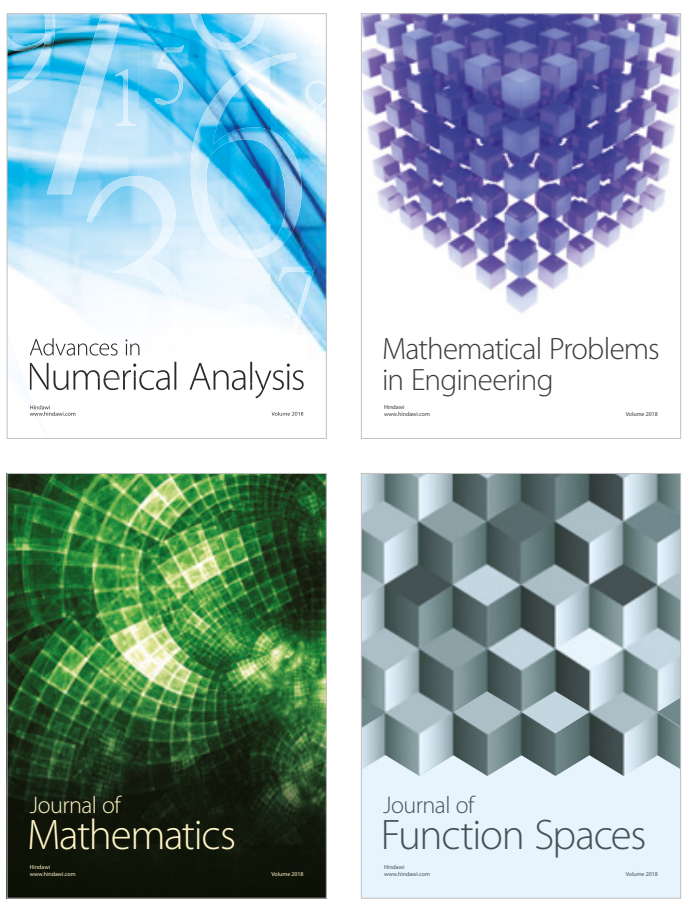

Mathematical Problems in Engineering

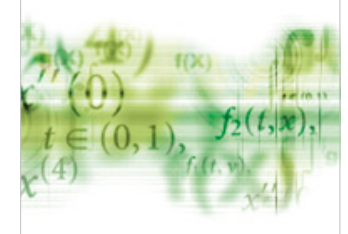

International Journal of

Differential Equations

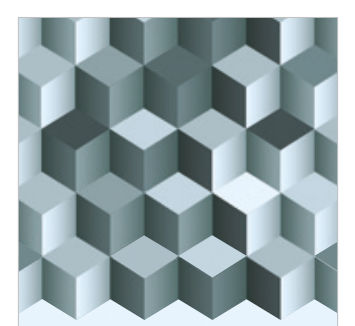

Journal of

Function Spaces

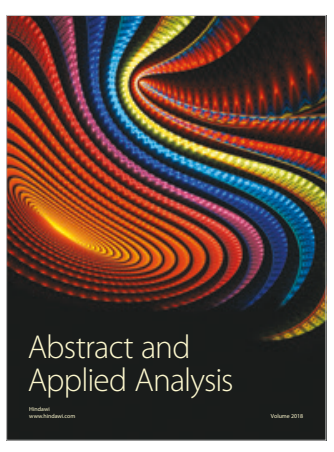

The Scientific

World Journal

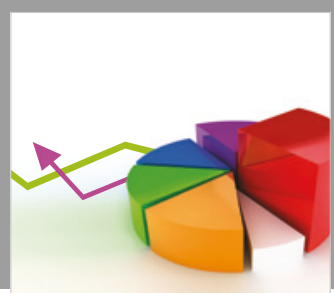

Journal of

Probability and Statistics
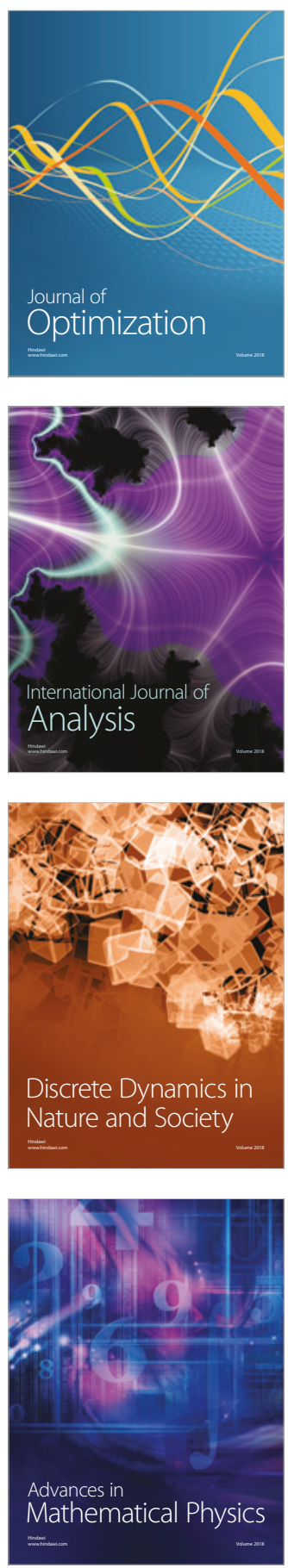